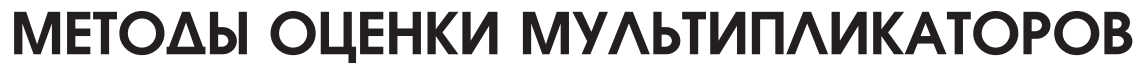 ВЫПУСКА В ОТСУТСТВИЕ ПОАНОЙ ТАБАИЦЫ “ЗАТРАТЫ - ВЫПУСК”
}

\section{C.A. Воропанов}

Воропанов Сергей Алексеевич - кандидат экономических наук, доцент. Российская академия народного хозяйства и государственной службы при Президенте Российской Федерации, Тольяттинский филиал, пр-т Степана Разина, 23, Тольятти, Россия, 445040. E-mail: voropanov.serega@yandex.ru.

В обзоре представлены относительно малоизвестные в отечественной литературе методы получения оценок мультипликаторов выпуска (мультипликаторов конечного спроса) в условиях минимума информации. В этих условиях исследователю приходится выбирать между тремя подходами: самостоятельно разрабатывать региональные базовые таблицы на основе широкомасштабного единовременного обследования - подход SBT (SurveyBased Tables); строить расчетные региональные таблицы на основе данных национальных (базовых или расчетных) таблиц - подход RIMS (Regional Input-Output Modeling System); ограничиться отдельными вычислениями, опирающимися на доступную в традиционной статистике информацию или на информацию, которая может быть получена из традиционной статистики при минимальной затрате ресурсов на ее обработку, - подход SM (Shortcut Methods) или методы минимума информации. Акцент сделан на SM-подходе: эволюции метода, критике исходных предпосылок, результатам эмпирической проверки.

Модель «затраты - выпуск», региональный межотраслевой анализ, мультипликаторы выпуска, мультипликаторы конечного спроса, методы оченивания мультипликаторов, SBT (Survey-Based Tables), RIMS (Regional Input-Output Modeling System), SM (Shortcut Methods).

DOI: $10.14530 /$ se.2015.4.148-164

Как на уровне национальных экономик, так и на региональном уровне модель В. Леонтьева «затраты - выпуск» (для отечественных экономистов более привычным является термин «межотраслевой баланс производства и распределения продукции») зарекомендовала себя эффективным инструментом проведения расчетов по анализу и прогнозу производства и распределения продукции, образования конечного спроса, формирования доходов, оценки занятости и др. Необходимым условием эффективного

(C) Воропанов С.А., 2015 
использования модели «затраты - выпуск» является наличие разрабатываемой государственными органами статистики информационной базы, включающей, во-первых, периодически, в среднем раз в пять лет, составляемые базовые ${ }^{1}$ таблицы «затраты - выпуск» по широкой номенклатуре отраслей - до нескольких сотен позиций, и, во-вторых, ежегодные расчетные таблицы, разрабатываемые по сокращенной номенклатуре отраслей до нескольких десятков позиций. Такая система существует в развитых странах, такая система существовала в СССР. Более того, в СССР базовые и расчетные балансы разрабатывались также в разрезе союзных республик и отдельных экономических районов. Аналогичные работы выполнялись Росстатом: построены таблицы РФ за 1995-2003 гг. по методологии системы национальных счетов (CHC), но без регионального разреза, ряд прервался из-за перехода статистики на международные классификаторы отраслей и продуктов.

Главная проблема использования метода «затраты - выпуск» на региональном (субрегиональном) уровне - отсутствие отчетной статистической базы. В этих условиях исследователю приходится выбирать между тремя подходами:

1. Самостоятельно разрабатывать региональные базовые таблицы на основе широкомасштабного единовременного обследования - подход SBT (Survey-Based Tables).

2. Строить расчетные региональные таблицы на основе данных национальных (базовых или расчетных) таблиц - подход RIMS (Regional InputOutput Modeling System).

3. Ограничиться отдельными вычислениями, опирающимися на доступную в традиционной статистике информацию или на информацию, которая может быть получена из традиционной статистики при минимальной затрате ресурсов на ее обработку, - подход SM (Shortcut Methods) или методы минимума информации [11].

Понятно, что идеальным является SBT-подход, позволяющий в итоге использовать весь наработанный арсенал методов межотраслевого анализа на основе таблиц «затраты - выпуск». В Российской Федерации этот подход реализован, например, при построении таблиц для Республики Башкортостан за 1995 г. в разрезе 227 отраслей [9]. Основной недостаток этого подхода - его высокая ресурсоемкость и невозможность реализации без серьезной поддержки местных властей.

\footnotetext{
1 Базовые таблицы составляются в год широкомасштабного единовременного обследования структуры затрат на производство товаров и услуг, проводимого по решению правительства. Агрегированные расчетные таблицы составляются ежегодно на основе информации, полученной из базовых таблиц и текущих значений окаймляющих итогов. В России последнее единовременное обследование проведено в 2011 г.
} 
RIMS-подход сводится в конечном итоге к получению проекции технологических коэффициентов ${ }^{1}$ национальной таблицы на региональную таким образом, чтобы обеспечить выход на заданные окаймляющие итоги региональной таблицы «затраты - выпуск» [32]. Балансировка региональной таблицы осуществляется за счет итерационных процедур типа метода RAS или его модификаций [1]. Подобные методы были использованы при построении таблиц для Ивановской области [10] и Дальнего Востока [8]. Аналогичные процедуры были применены при построении в единой методологии расчетных национальных таблиц «затраты - выпуск» для РФ за 2003-й и последующие годы [1-3].

Методологию RIMS используе Бюро экономического анализа Министерства торговли США для построения региональных таблиц «затраты выпуск» с последующим расчетом на основе обратной матрицы Леонтьева различного рода мультипликаторов и известна под названием RIMS II [32]. Заказчиками расчетов с использованием RIMS II выступают как государственные, так и частные структуры.

RIMS-подход, обеспечивая меньшую точность по сравнению с SBT, требует для практической реализации существенно меньших затрат сил и средств. При этом, как и при SBT-подходе, итоговая таблица позволяет осуществлять весь комплекс расчетов по методу «затраты - выпуск», включающий, в частности, расчет мультипликаторов выпуска, доходов и занятости. Точность таких расчетов, естественно, будет ниже, чем при реализации SBT-подхода.

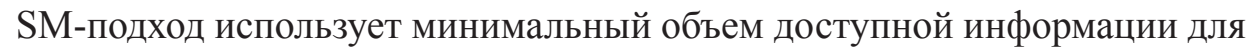
расчета различного рода мультипликаторов. К этой информации относятся, прежде всего, данные о материалоемкости (доле материальных затрат в составе валового выпуска отрасли ${ }^{2}$ ) в разрезе отраслей, выделенных в таблице, а также некоторая другая информация.

Рассмотрим относительно малоизвестные в отечественной литературе основные результаты, полученные к настоящему времени в рамках SМ-подхода.

Введем обозначения и формализуем задачу. Симметричная таблица «затраты - выпуск» представлена уравнением

$$
x=A x+f,
$$

где $x-n$-мерный вектор валовых выпусков, $f-n$-мерный вектор конечного спроса, $A=\left(a_{i j}\right), i, j=1, \ldots, n-$ технологическая матрица, коэффициенты

${ }^{1}$ В отечественной литературе более привычен термин «коэффициенты прямых материальных затрат».

${ }^{2}$ В методологии СНС это понятие соответствует удельному весу промежуточного потребления в стоимости валового выпуска отрасли. 
которой $a_{i j} \geq 0$ равняются удельным затратам продукции отрасли $i$ на производство единицы валового выпуска продукции отрасли $j, n-$ количество выделенных в таблице «затраты - выпуск» отраслей.

Обратная матрица Леонтьева (Leontief Inverse Matrix) $Q=\left(q_{i j}\right)$ рассчитывается по известной формуле $Q=(I-A)^{-1}$, где $I-$ единичная матрица.

Вектор простых мультипликаторов выпуска ${ }^{1}$ (Simple Output Multipliers, Final Demand Multipliers) $\mu=\left(\mu_{1}, \ldots, \mu_{n}\right)$ по определению равняется сумме по столбцу элементов обратной матрицы Леонтьева $\mu_{j}=\sum_{i=1}^{n} q_{i j}$, $j=1, \ldots, n$. Мультипликатор ${ }^{2} \mu_{j}$ характеризует суммарный рост валовых выпусков всех отраслей при увеличении конечного спроса отрасли $j$ на единицу [22, ch. 6.2.1].

Знание мультипликатора позволяет в условиях минимума доступной информации решать такого рода задачи: в какой отрасли инвестиции дадут максимальный рост совокупных валовых выпусков всех отраслей? В какой отрасли государственные закупки дадут максимальный эффект по критерию максимизации суммарных выпусков всех отраслей?

Рядом авторов предложены формулы, позволяющие оценить вектор мультипликаторов $\mu$ в условиях неполной информации, когда вся технологическая матрица $A$ неизвестна, а доступны только ее отдельные характеристики. К таким характеристикам относится, прежде всего, вектор отраслевых материалоемкостей (доли материальных затрат в валовом выпуске отрасли или, что то же самое, суммы технологических коэффициентов по столбцам)

$$
w_{j}=\sum_{i=1}^{n} a_{i j}<1, j=1, \ldots, n .
$$

Заметим, что условие (2) является достаточным для существования единственного неотрицательного решения уравнения (1) для любого неотрицательного вектора конечного спроса. Этот общеизвестный факт следует из возможности представления обратной матрицы Леонтьева сходящимся степенным рядом

$$
Q=(I-A)^{-1}=\sum_{i=0}^{\infty} A^{i}
$$

По-видимому, к первым работам³ в области оценки мультипликаторов выпуска в условиях минимума информации относятся работы Д. Бромли [13] и Д. Сальседо [33]. На основе экспериментов Д. Бромли установлена тесная статистическая связь между значениями $\mu_{j}$ и $w_{j}$, сама оценка $\mu_{j}$ не

\footnotetext{
${ }^{1}$ Иногда используется термин «мультипликатор конечного спроса».

2 Далее в работе для краткости будем использовать термин «мультипликатор».

3 Здесь и далее в статье автор не рассматривает вопросы приоритетности полученных различными исследователями результатов.
} 
проводилась. Д. Сальседо установлена тесная взаимосвязь между показателями $\mu_{j}$ и $\left(1-w_{j}\right)$.

Наконец, П.Е. Нельсон и Дж.С. Перрен [29, с. 91] пришли к выводу, что SM-подход (методы минимума информации) «может дать значения мультипликаторов, которые довольно хорошо согласуются с результатами, полученными из традиционного анализа «затраты - выпуск», основанного на расчете обратной матрицы Леонтьева (в предложенной выше терминологии это SBT- и RIMS-подходы). В указанных исследованиях использовалась техника статистического (регрессионного) анализа.

Р.Л. Дрейк [20] для оценки мультипликатора разложил его на три составляющих:

а) начальный эффект, равный 1 ;

б) прямой (direct) эффект, равный $w_{j}$;

в) косвенный (indirect) эффект, равный $\mu_{j}-\left(1+w_{j}\right)$.

Используя предположение о линейной связи косвенного и прямого эффекта и технику регрессионного анализа, Р.Л. Дрейк в конечном итоге получил следующую зависимость:

$$
\mu_{j}=1+w_{j} /(1-\bar{w}), j=1, \ldots, n,
$$

где $\bar{w}=n^{-1} \sum_{i=1}^{n} w_{i}-$ средняя арифметическая из отраслевых материалоемкостей.

Назовем формулу (3) базовой формулой или базовой оценкой мультипликатора, т. к. в ней используется минимально доступная информация о технологической матрице $A$.

Используя метод случайных матриц (Random Matrix Approach), Р.Л. Бурфорд и Дж.Л. Кац [16] получили идентичный базовой формуле (3) результат оценки мультипликатора (см. также более ранние работы этих авторов [14; $15 ; 17 ; 18])$.

Далее в публикациях ряда исследователей были предприняты попытки улучшить базовую оценку (3) с использованием дополнительной информации о матрице $A$. В этих же публикациях развернулась дискуссия по теоретическому обоснованию и экспериментальной оценке точности предложенных методов.

Например, Р.Л. Бурфордом и Дж.Л. Кацем получена оценка [16] мультипликаторов в предположении, что кроме сумм по столбцам известны также суммы технологических коэффициентов по строкам $r_{i}=\sum_{j=1}^{n} a_{i j}$ матрицы $A$.

$$
\mu_{j}=1+w_{j} /\left(1+\frac{\sum_{i=1}^{n} r_{i} w_{i}}{\sum_{i=1}^{n} r_{i}}\right), j=1, \ldots, n .
$$


Заметим, что теоретически такая оценка мультипликатора вероятно допустима и даже обладает известной математической красотой, но сумма технологических коэффициентов по строке - экономическая бессмыслица: нельзя складывать разнородные удельные показатели по строке (в отличие от суммирования межотраслевых потоков по столбцу технологической матрицы).

В этой же работе [16] получена оценка для $j$-й компоненты вектора мультипликаторов, исходя из предположения о том, что кроме вектора $w_{j}$ известен еще и столбец $j$ (и только он один) матрицы $A^{1}$.

$$
\mu_{j}=1+\left(w_{j}+P_{j} / S_{j}\right) /\left(1-a_{j j}-P_{j} S_{j} / n\right), j=1, \ldots, n,
$$

где $P_{j}=\sum_{\substack{i=1 \\ i \neq j}}^{n} a_{i j} w_{j}, S_{j}=1-\sum_{\substack{i=1 \\ i \neq j}}^{n} w_{i} / n$.

Такой подход, очевидно, приемлем для отраслей с относительно простой структурой материальных затрат или для отраслей, структура материальных затрат которых может быть оценена, исходя из данных традиционной статистической отчетности.

П.Дж. Фибс и А.Дж. Холсман [30; 31] получили красивую оценку мультипликаторов в предположении, что кроме вектора материалоемкости известны все диагональные коэффициенты $a_{j j}$ матрицы $A$.

$$
\mu_{i}=\frac{1}{1-a_{i i}}\left[1+\left(w_{i}-a_{i i}\right) / \prod_{\substack{j=1 \\ j \neq i}}^{n}\left(1-a_{j j}\right)\right], i=1, \ldots, n .
$$

При тех же предположениях (известны вектор материалоемкости и все диагональные коэффициенты технологической матрицы $A$ ) Дж.Л. Кацем [25] была предложена новая формула для оценки мультипликаторов ${ }^{2}$. В этой же работе проведены сравнительные расчеты на пяти среднеразмерных таблицах штатов США (от 28 до 51 выделенных в таблицах отраслей) и шести малоразмерных (11 отраслей) таблиц для регионов Австралии. Экспериментальные расчеты показали, что формула (6) весьма неточна и, вероятно, пригодна лишь для небольших регионов с малым количеством выделенных в таблице «затраты - выпуск» отраслей (ошибки в пределах 0,6-9,8\% по формуле (6) в сравнении с ошибками в 0,8-2,2\% по формуле Дж.Л. Каца). Но когда количество выделенных в таблице «затраты - выпуск» отраслей составляло несколько десятков (30-50 отраслей), точность формулы (6) при оценке мультипликаторов резко снижалась (ошибки в 11,4-56,8\%, а для штата Вашингтон 150,7\% (!) по формуле (6), при ошибках всего в 0,5-2,3\% по формуле Дж.Л. Каца).

1 Формула (5) в целях компактности приведена в несколько отличном от оригинала виде.

2 Эта формула не приводится ввиду ее громоздкости. 
При доказательствах формул (3)-(5) Р.Л. Бурфорд и Дж.Л. Кац использовали метод случайных матриц. Оценка мультипликатора получена ими на основе оценки математического ожидания мультипликатора на множестве случайных матриц $A$ с заданными итогами по столбцам при предположении о равномерном распределении технологических коэффициентов матрицы $A$ внутри ее столбцов [16].

Предположение очень сильное и явно не соотносящееся с действительностью. На практике зачастую в каждом столбце $j$ диагональный коэффициент $a_{j j}$ является максимальным (по крайней мере для высокоагрегированных матриц), более того, ряд технологических коэффициентов apriori равны нулю.

На последнее обстоятельство указывает Ф.Дж. Хэрриган [21], а также Дж.Л. Кац и Р.Л. Бурфорд в ответной публикации [28]. Ф.Дж. Харриган предложил для оценки распределения коэффициентов матрицы $A$ применять таблицы-аналоги, в качестве которых могут фигурировать таблицы данного региона за предшествующие годы, таблицы других регионов со схожей структурой экономики или страны в целом. Эмпирические расчеты, проведенные на материалах 46-отраслевой таблицы «затраты - выпуск» Шотландии, дали более точные в сравнении с базовой формулой (3) результаты. Средняя ошибка по базовой формуле составила 22,2\% против 14,8\% при альтернативном подходе. Впрочем, и ошибка в 14,8\% особенно не вдохновляет. При расчетах в качестве таблицы-аналога выступала таблица Великобритании.

Р. Дженсен и Дж. Хевингс в работе с весьма остроумным названием [23] подвергли Р.Л. Бурфорда, Дж.Л. Каца и других сторонников SM-подхода жесткой критике (см. также ответ Р.Л. Бурфорда и Дж.Л. Каца с не менее остроумным названием [19] и последовавший комментарий к нему [24]).

В первую очередь критиковалось предположение о том, что распределение коэффициентов в столбцах матрицы играет относительно небольшую роль в определении конкретных значений мультипликатора, в то время как мультипликаторы имеют тенденцию быть зависимыми от общей структуры коэффициента в столбцах. Р.Л. Бурфорд и Дж.Л. Кац утверждают, что материалоемкость является ключевым фактором, определяющим величины мультипликатора, это означает, что причинно-следственная связь в формировании мультипликатора начинается с отраслевых материалоемкостей. Однако из логики модели «затраты - выпуск», следует, что $w_{j}=\sum_{i=1}^{n} a_{i j}$ просто сумма по столбцам технологических коэффициентов и сама по себе не играет никакой роли в процессе формирования мультипликатора через обычные процедуры расчета обратной матрицы Леонтьева [23, с. 755]. И с этими утверждениями в теоретико-методологическом плане нельзя не согласиться. В частности, выше уже указывалось на несоответствие практике 
предположения о равномерном распределении технологических коэффициентов матрицы $A$ внутри ее столбцов.

Далее Р. Дженсен и Дж. Хевингс пишут, что работы по оценке мультипликаторов не представляют практически никаких новых или оригинальных концепций и являются по существу идеей, которая была с нами в течение нескольких десятилетий... в оперативном смысле ничего нового не было добавлено в арсенал регионального анализа [23, с. 756]. Действительно, базовая оценка (3) известна достаточно давно, однако, как показано выше, Р.Л. Бурфордом, Дж.Л. Кацем и другими исследователями получены новые оценки для мультипликаторов при использовании дополнительной информации о матрице $A$.

Затем Р. Дженсен и Дж. Хевингс утверждают, что если интерес ориентирован только на общий эффект, то формулы Р.Л. Бурфорда - Дж.Л. Каца ${ }^{1}$ будут работать так же хорошо, как и многие другие. С другой стороны, если интерес ориентирован на прямое и косвенное воздействие спроса на отдельные сектора экономики, то формулы Р.Л. Бурфорда - Дж.Л. Каца предоставляют мало удобств, и нет уверенности, что оценки будут приемлемо точными. Отражение отраслевого распределения эффектов, рассчитанных на основе модели «затраты - выпуск», является смыслом существования этих моделей. Подход Р.Л. Бурфорда - Дж.Л. Каца явно недостаточен [23, с. 756]. Это замечание не вполне корректное, нельзя требовать от модели результатов, для получения которых она не предназначена. Это то же самое, как упрекать Дж.М. Кейнса в том, что его мультипликатор не отражает отраслевые эффекты.

Формально Р. Дженсен и Дж. Хевингс правы, утверждая, что когда суммы по столбцам и по строкам являются доступными, то техника Р.Л. Бурфорда - Дж.Л. Каца и П.Дж. Фибса - А.Дж. Холсмана, при которой известны технологические коэффициенты для отдельных столбцов, является частными случаями более общего решения RAS-метода [23, с. 756-757]. Это не совсем точное замечание, ибо в формулах Р.Л. Бурфорда - Дж.Л. Каца и П.Дж. Фибса - А.Дж. Холсмана предполагается, что известен один из столбцов матрицы $A$, тогда только для этой отрасли оценка мультипликатора может быть улучшена (см., например, формулу (5). RAS-метод (и его модификации) [1] предполагает, что известно начальное приближение матрицы $A$, т. е., как минимум, «важные коэффициенты» ${ }^{2}$ или их начальное приближение заранее известны, причем коэффициенты эти расположены в разных столбцах технологической матрицы. В оценках Р.Л. Бурфорда -

\footnotetext{
В оригинальном тексте формула Бурфорда - Каца - ВК (примеч. авт.).

2 «Важные коэффициенты»- коэффициенты матрицы $A$, оказывающие наибольшее влияние на точность решения уравнения (1).
} 
Дж.Л. Каца и П.Дж. Фибса - А.Дж. Холсмана такая информация не востребована, за исключением оценок, использующих знание диагональных коэффициентов матрицы $A$, как, например, в формуле (6).

Действительно, данные о структуре затрат (столбец матрицы $A$ ) менее доступны, чем ее итоги по столбцам (отраслевые материалоемкости). Но для отдельных таблиц отдельных регионов такие данные могут быть получены. Например, для малоразмерных таблиц небольших регионов, где данная отрасль представлена единственным продуктом, производящимся по единственной технологии с относительно простой структурой затрат.

Более того, верно и такое утверждение: для таблиц большой размерности, когда понятие «отрасль» практически тождественно понятию «продукт», и продукт производится по единственной технологии ${ }^{1}$, для отдельных отраслей возможно получить достаточно точные оценки структуры их затрат.

Вполне обоснованно критикуя исходные посылки Р.Л. Бурфорда и Дж.Л. Каца и других сторонников SM-подхода, Р. Дженсен и Дж. Хевингс переносят критику и на конечный результат. Между тем история науки знает случаи, когда неверные исходные предпосылки давали в конечном итоге верный результат.

В инженерной науке часто возникает метод, который обосновывается практическими ситуациями, но получает строго математическое обоснование намного позже. Например, открытие О. Хевисайдом операционного исчисления [6].

Дискуссия Р. Дженсена - Дж. Хевингса и Р.Л. Бурфорда - Дж.Л. Каца о приемлемости SM-подхода очень напоминает нескончаемый и не могущий быть законченным по определению спор сторонников технического и фундаментального анализа при прогнозировании рынка ценных бумаг.

Главный и окончательный аргумент в пользу приемлемости SM-подхода - практика. В процитированных статьях различными авторами для различных стран, регионов, на таблицах различной размерности и за разные годы проведены многочисленные эксперименты по оценке точности $\mathrm{SM}$-подхода. В подавляющем большинстве получена, в том числе и автором, вполне приемлемая точность оценки мультипликаторов.

Автором настоящего обзора на основе методов теории положительных операторов [6] получены двухсторонние оценки мультипликаторов выпуска [4]:

$$
1+w_{j} /\left(1-\min _{j} w_{j}\right) \leq \mu_{j} \leq 1+w_{j} /\left(1-\max _{j} w_{j}\right), j=1, \ldots, n
$$

\footnotetext{
1 Кстати, перечисленное и есть классические аксиомы модели В. Леонтьева.
} 
Так как неравенства (7) задают отрезок, внутри которого находится истинное значение мультипликатора, то в частном случае в качестве точечной оценки можно использовать формулу

$$
w_{j} \approx 1+w_{j} /(1-\bar{w}), j=1, \ldots, n,
$$

что дает другим способом доказанный ранее результат (3).

В случае, если известен $j$-й столбец матрицы $A$, и только он один, то для отрасли $j$ двухсторонняя оценка мультипликатора была улучшена автором [4, с. 97]:

$$
\begin{gathered}
1+w_{j}+\sum_{i=1}^{n} a_{i j} w_{i} /\left(1-\min _{j} w_{j}\right) \leq \mu_{j} \leq 1+w_{j}+\sum_{i=1}^{n} a_{i j} w_{i} /\left(1-\max _{j} w_{j}\right), \\
\text { для всех } j=1, \ldots, n .
\end{gathered}
$$

По аналогии с (8) в качестве точечной оценки мультипликатора может быть использована формула

$$
\mu_{j} \approx 1+w_{j}+\sum_{i=1}^{n} a_{i j} w_{i} /(1-\bar{w}), j=1, \ldots, n .
$$

Очевидно, что использование соотношений (9) и (10) для оценки j-й компоненты мультипликатора выпуска возможно лишь в случае, когда известен хотя бы один столбец $j$ матрицы $A$.

Также было предложено [4, с. 97] искать оценки мультипликатора $\mu_{j}$ внутри отрезка, задаваемого уравнением

$$
\begin{gathered}
\mu_{j} \approx \beta_{j} \bar{\mu}_{j}+\left(1-\beta_{j}\right) \overline{\bar{\mu}}_{j}, j=1, \ldots, n, \\
\bar{\mu}_{j} \leq \mu_{j} \leq \overline{\bar{\mu}}_{j}, j=1, \ldots, n,
\end{gathered}
$$

где $\bar{\mu}_{j}\left(\overline{\bar{\mu}}_{j}\right)-$ соответственно нижняя (верхняя) оценки $\mu_{j}$, определяемые в зависимости от доступной информации формулами (7) или (9), $0 \leq \beta_{j} \leq 1$. При $\beta_{j}=0\left(\beta_{j}=1\right)$ имеем крайние точки отрезка.

Для оценки значений коэффициентов $\beta_{j}$ используется межотраслевая таблица-аналог. В качестве последней можно использовать таблицы «затраты - выпуск» данного региона за предшествующие годы, таблицы других регионов со схожей структурой экономики или страны в целом.

При доказательствах формул (7)-(10) автором вообще не использовалось предположение о распределении технологических коэффициентов, которое подверглось выше критике. Были использованы только стандартные предположения модели Леонтьева относительно технологических коэффициентов: $a_{i j} \geq 0, \sum_{i=1}^{n} a_{i j}<1$ для всех $i, j=1, \ldots, n$. Таким образом, автором еще раз, используя другой математический инструментарий, была подтверждена верность оценки (3). Причем внутренняя структура затрат отраслей, как ни странно, никак непосредственно не влияет на получение оценок (8). Строгие доказательства формул (7)-(12) приведены в работе [5]. 
Проведенные автором обзора вычислительные эксперименты на таблицах «затраты - выпуск» Германии и Франции в текущих основных ценах за пятнадцатилетний период с 1995 по 2009 г. для 34-отраслевой номенклатуры ${ }^{1}$ дали вполне приемлемые результаты [5]. Базовая формула (3) дает хорошие результаты в оценке мультипликаторов выпуска. Средняя абсолютная ошибка по всем отраслям за все годы составила для Германии 1,69\%, для Франции 1,08\%. Дополнительная информация о структуре затрат отрасли (формулы (5), (10)) улучшает среднюю оценку в 2-4 раза. Дополнительная информация о матрице предыдущего года улучшает оценку мультипликаторов приблизительно в 3-4 раза [5].

С середины 1980-х гг. интерес к оценкам мультипликаторов на основе

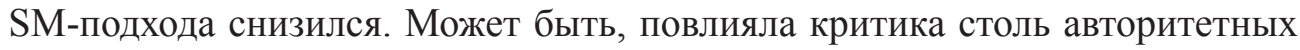
в области моделей «затраты - выпуск» специалистов, как Р. Дженсен и Дж. Хевингс, но в последующие годы существенных теоретических результатов, улучшающих ранее доказанные оценки для мультипликаторов, не получено. Последняя известная работа Р.Л. Бурфорда и Дж.Л. Каца относится к 1985 г. [19], повторяет ранее полученные оценки мультипликаторов выпуска и распространяет их подход для оценок других видов мультипликаторов $^{2}$. В основном исследователи занимались эмпирическими расчетами (перечень работ см. в [12]).

Главная проблема при оценке точности расчета мультипликаторов заключается в том, что, по-видимому, не существует математически строгого доказательства, что тот или иной из рассмотренных методов apriori всегда точнее другого. Следовательно, любые даже многократные эмпирические исследования по оценке точности методов расчета мультипликаторов могут только подтвердить, что для данной страны (региона) за анализируемый период данный метод оказался точнее другого. Но из этого совсем не следует, что в другой ситуации, для других стран, за другие годы результат не изменится. Единственное к настоящему моменту известное нам исключение доказано [4, с. 96-97], что отрезок, задаваемый неравенствами (9), всегда лежит внутри отрезка, задаваемого неравенствами (7). Соответственно, интервальные оценки, задаваемые неравенствами (9), в среднем будут лучше оценок, задаваемых неравенствами (7).

Проведенный анализ позволяет представить существующие методы оценки мультипликаторов в виде классификации с точки зрения математического инструментария и основных гипотез, лежащих в основе (табл.).

${ }^{1}$ Из стандартной 35-отраслевой номенклатуры, используемой в проекте Международной ассоциации «затраты - выпуск» (International Input-Output Association), была исключена отрасль «домашнее хозяйство», т. к. для всех таблиц и строка, и столбец по данной отрасли были нулевыми.

2 Отметим, что предложенный автором обзора подход легко позволяет получить двухсторонние оценки для мультипликаторов занятости, доходов и т. д. 
Математический инструментарий и основные гипотезы, лежащие в основании различных методов оценки мультипликаторов

\begin{tabular}{|c|c|c|c|c|}
\hline $\begin{array}{l}\text { № } \\
\Pi / \Pi\end{array}$ & Метод & Авторы & $\begin{array}{l}\text { Математический } \\
\text { инструментарий }\end{array}$ & Основные гипотезы \\
\hline 1 & \begin{tabular}{|l|} 
SВТ-подход \\
(Survey- \\
Based Tables)
\end{tabular} & В. Леонтьев & $\begin{array}{l}\text { Сплошное обсле- } \\
\text { дование }\end{array}$ & $\begin{array}{l}\text { Линейная зависимость меж- } \\
\text { отраслевых потоков от валового } \\
\text { выпуска отрасли потребителя }\end{array}$ \\
\hline 2 & $\begin{array}{l}\text { RIMS-подход } \\
\text { (Regional } \\
\text { Input-Output } \\
\text { Modeling } \\
\text { System) } \\
\end{array}$ & $\begin{array}{l}\text { Бюро экономиче- } \\
\text { ского анализа Ми- } \\
\text { нистерства торговли } \\
\text { США [32] }\end{array}$ & $\begin{array}{l}\text { Построение } \\
\text { региональной } \\
\text { матрицы на осно- } \\
\text { ве национальной } \\
\text { таблицы }\end{array}$ & $\begin{array}{l}\text { Подобие национальных и регио- } \\
\text { нальных межотраслевых таблиц }\end{array}$ \\
\hline \multirow{3}{*}{3} & \multirow{3}{*}{$\begin{array}{l}\text { SM-подход } \\
\text { (Shortcut } \\
\text { Methods) }\end{array}$} & $\begin{array}{l}\text { Р.Л. Бурфорд и } \\
\text { Дж.Л. Кац [14-19; } \\
\text { 25-28], П.Дж. Фибс } \\
\text { и А.Дж. Холсман } \\
\text { [30; 31], Ф.Дж. Хар- } \\
\text { риган [21] }\end{array}$ & $\begin{array}{l}\text { Метод случай- } \\
\text { ных матриц } \\
\text { (Random Matrix } \\
\text { Approach) }\end{array}$ & $\begin{array}{l}\text { Предполагается равномерность } \\
\text { распределения технологических } \\
\text { коэффициентов } \\
\text { Ф.Дж. Харриган предложил для } \\
\text { оценки распределения коэффи- } \\
\text { циентов матрицы } A \text { применять } \\
\text { таблицы-аналоги }\end{array}$ \\
\hline & & $\begin{array}{l}\text { Д. Бромли [13], } \\
\text { Д. Сальседо [33], } \\
\text { П.Е. Нельсон и } \\
\text { Дж.С. Перрен [29] }\end{array}$ & $\begin{array}{l}\text { Техника ста- } \\
\text { тистического } \\
\text { (регрессионного) } \\
\text { анализа }\end{array}$ & $\begin{array}{l}\text { Эмпирический подход, основан- } \\
\text { ный на том, что существует ста- } \\
\text { тистически значимая связь меж- } \\
\text { ду материалоемкостью отраслей } \\
\text { и мультипликаторами выпуска }\end{array}$ \\
\hline & & $\begin{array}{l}\text { С.А. Воропанов } \\
{[4 ; 5]}\end{array}$ & $\begin{array}{l}\text { Теория положи- } \\
\text { тельных операто- } \\
\text { ров (операторов } \\
\text { в пространствах } \\
\text { с конусом) } \\
\end{array}$ & Строгая математическая теория \\
\hline
\end{tabular}

Источник: составлено автором.

В заключение отметим, что автор, во-первых, не ставил вопрос о приоритетности полученных различными исследователями результатов; во-вторых, не претендует на окончательную полноту обзора; в-третьих, не рассматривает оценки для других видов мультипликаторов, таких как мультипликаторы занятости и доходов; в-четвертых, самостоятельно осуществил перевод оригинальных статей, рассмотренных в обзоре, стремясь не к буквальной точности перевода, а скорее, к точности передачи мысли, изложенной в цитируемом источнике.

\section{Благодарности}

Автор признателен д-ру экон. наук, проф. Э.Ф. Баранову (НИУ «Высшая школа экономики», Москва), д-ру экон. наук А.Г. Коровкину (Институт народнохозяйственного прогнозирования РАН, Москва) и проф. В.М. Абрамову (Prof. V.M. Abramov, Swinburne University of Technology, Australia) за ценные советы при подготовке настоящего обзора. 


\section{СПИСОК ЛИТЕРАТУРЫ}

1. Баранов Э.Ф., Ким И.А., Пионтковский Д.И., Старицына Е.А. Вопросы построения таблиц «затраты - выпуск» России в международных классификаторах // Экономический журнал ВШЭ. 2014. № 1. С. 7-41.

2. Баранов Э.Ф., Ким И.А., Пионтковский Д.И., Старицьна Е.А. Методологические проблемы построения систем таблиц «затраты - выпуск» России в классификаторах отраслей и продуктов, соответствующих международным стандартам. Препринт WP2/2013.06. М.: Высшая школа экономики. 2013. 41 с.

3. Баранов Э.Ф., Ким И.А., Старицына Е.А. Методологические вопросы реконструкции системы таблиц «затраты - выпуск» России за 2003 и последующие годы в структуре ОКВЭД-ОКПД // Вопросы статистики. 2011. № 12. С. 3-8.

4. Воропанов С.А. Оценка некоторых характеристик модели Леонтьева в условиях неполной информации // Естественные и математические науки в современном мире: сборник статей по материалам IX-X международной научно-практической конференции. Новосибирск: Изд-во «СибАК», 2013. № 10-11. С. 92-98.

5. Воропанов C.A. Применение метода двухстороннего оценивания мультипликаторов выпуска в условиях отсутствия полной таблицы «затраты - выпуск» (на примере статистики Германии и Франции) // Проблемы прогнозирования. 2015. № 4. С. 131-141.

6. Красносельский М.А., Вайникко Г.М., Забрейко П.П., Рутиикий Я.Б., Стеиенко В.Я. Приближенное решение операторных уравнений. М.: Наука, 1969. 456 с.

7. Математическая энциклопедия. URL: http://dic.academic.ru/dic.nsf/enc mathematics/3696/ОПЕРАЦИОННОЕ (дата обращения: 17.04.2014).

8. Михеева Н.Н. Статистическая оценка таблиц «затраты - выпуск» для российского Дальнего Востока // Пространственная экономика. 2005. № 2. С. 61-79.

9. Нигматулин Р.И., Саяпова А.Р., Мазитова Л.Д. Таблицы «затраты - выпуск» Республики Башкортостан // ЭКО. 2006. № 3. С. 90-105.

10. Серебряков Г.Р., Узяков М.Н., Янтовский А.А. Межотраслевая модель экономики Ивановской области // Проблемы прогнозирования. 2002. № 5. С. 64-74.

11. Abramov V.M., Voropanov S.A. Regional Analysis and Forecast under Limited Available Information // Проблемы и перспективы развития банковской системы Республики Таджикистан в условиях неустойчивости состояния мировых финансов: материалы республиканской научно-практической конференции (Душанбе, 2014, 1516 мая). Душанбе, 2014. С. 133-135.

12. Bekhet H.A. Output, Income and Employment Multipliers in Malaysian Economy: Input-Output Approach // International Business Research. 2011. Vol. 4. No. 1. Pp. 208223. DOI: $10.5539 /$ ibr.v4n1p208.

13. Bromley D.W. An Alternative to Input-Output Models: A Methodological Hypothesis // Land Economics. 1972. Vol. 48. Pp. 125-133. DOI: 10.2307/3145471.

14. Burford R.L., Katz J.L. Regional Input-Output Multipliers without a Full I-O Table: Reply // Annals of Regional Science. 1978. Vol. 12. No. 3. Pp. 99-102. DOI: 10.1007/ BF01286127.

15. Burford R.L. Regional Input-Output Multipliers without a Full I-O Table // Annals of Regional Science. 1977. Vol. 11. No. 3. Pp. 21-38. DOI: 10.1007/BF01287766.

16. Burford R.L., Katz J.L. A Method of Estimation of Input-Output Type Output Multipliers when no I-O Model Exists // Journal of Regional Science. 1981. Vol. 21. No. 2. DOI: 10.1111/j.1467-9787.1981.tb00691.x.

17. Burford R.L., Katz J.L. An Estimator of Regional Interindustry Multipliers without an I-O Matrix // Business and Economics Section Proceedings of the American Statistical Association. 1977. Pp. 453-458. 
18. Burford R.L., Katz J.L. Regional Input-Output Multipliers without a Full I-O Table: (Communication) // Annals of Regional Science. 1978. Vol. 12. No. 2. Pp. 105-106. DOI: 10.1007/BF01286115.

19. Burford R.L., Katz J.L. Shortcut Input-Output Multipliers, Alive and Well: Response to Jensen and Hewings // Environment and Planning A. 1985. Vol. 17. No. 11. Pp. 15411549. DOI: $10.1068 / \mathrm{a} 171541$.

20. Drake R.L. A Short Cut to Estimates of Regional Input-Output Multipliers: Methodology and Evaluation // International Regional Science Review. 1976. Vol. 1. No. 2. Pp. 1-17. DOI: 10.1177/016001767600100201.

21. Harrigan F.J. The Estimation of Input-Output Type Output Multipliers when no Input-Output Model Exists: A Comment // Journal of Regional Science. 1982. Vol. 22. No. 3. Pp. 375-381. DOI: 10.1111/j.1467-9787.1982.tb00762.x.

22. Input-Output Analysis: Foundations and Extensions. Second Edition / Edited by R.E. Miller, P.D. Blair. Cambridge University Press, 2009. 784 p. DOI: 10.1017/ CBO9780511626982.

23. Jensen R.C., Hewings G.J.D. Shortcut Input-Output Multipliers: A Requiem // Environment and Planning A. 1985. Vol. 17. No. 6. Pp. 747-759. DOI: 10.1068/a170747.

24. Jensen R.C., Hewings G.J.D. Shortcut Input-Output Multipliers: The Resurrection Problem (a Reply) // Environment and Planning A. 1985. Vol. 17. No. 11. Pp. 1551-1552. DOI: $10.1068 /$ a171551.

25. Katz J.L. A Shortcut Method for Computing Final Demand Multipliers for Small Regions: Comment // Environment and Planning A. 1983. Vol. 15. No. 4. Pp. 551-554. DOI: $10.1068 / \mathrm{a} 150551$.

26. Katz J.L., Burford R.L. A Comparison of Estimators of Output Multipliers from Incomplete Input-Output Data // The Annals of Regional Science 1981. Vol. 15. No. 2. Pp. 39-54. DOI: 10.1007/BF01287438.

27. Katz J.L., Burford R.L. Shortcut Formulas for Output, Income and Employment Multipliers // The Annals of Regional Science. 1985. Vol. 19. No. 2. Pp. 61-76. DOI: 10.1007/BF01287578.

28. Katz J.L., Burford R.L. The Estimation of Input-Output Type Output Multipliers when no Input-Output Model Exists: A Reply // Journal of Regional Science. 1982. Vol. 22. No. 3. Pp. 383-387. DOI: 10.1111/j.1467-9787.1982.tb00763.x.

29. Nelson P.E., Perrin J.S. A Short Cut for Computing Final Demand Multipliers: Some Empirical Results // Land Economics. 1978. Vol. 54. No. 1. Pp. 82-91. DOI: $10.2307 / 3146204$.

30. Phibbs P.J., Holsman A.J. A Reply to Katz's Comment / Letter to the Editor // Environment and Planning A. 1983. Vol. 15. No. 4. Pp. 555-558. DOI: 10.1068/a150555.

31. Phibbs P.J., Holsman A.J. A Shortcut Method for Computing Final Demand Multipliers for Small Regions // Environment and Planning A. 1980. Vol. 12. No. 9. Pp. 1001-1008. DOI: 10.1068/a121001.

32. Regional Input-Output Modeling System (RIMS II). URL: https://www.bea.gov/ regional/rims/ (дата обращения: 15.04.2014).

33. Salcedo D. A Short Cut Method for Determining the Impact of an Economic Activity on an Entire Economy. Unpublished Report Office of Information Services. Texas, 1972. Цит. по: Jensen R.C., Hewings G.J.D. Shortcut Input-Output Multipliers: A Requiem // Environment and Planning A. 1985. Vol. 17. No. 6. Pp. 747-759. DOI: 10.1068/a170747.

34. The World Input-Output Database (WIOD): Contents, Sources and Methods / Edited by M.P. Timmer. WIOD. Working Paper. No. 10. 2012. URL: http://www.wiod.org/ publications/papers/wiod10.pdf (дата обращения: 06.11.2013). 


\title{
METHODS OF VALUATION OF OUTPUT MULTIPLIERS' IN THE ABSENCE OF COMPLETE INPUT-OUTPUT TABLE
}

\begin{abstract}
S.A. Voropanov
Voropanov Sergey Alekseevich - PhD in Economics, Associate Professor. Russian Presidential Academy of National Economy and Public Administration, Tolyatti Branch; 23 pr-t Stepana Razina, Tolyatti, Samarskaya Oblast, Russia, 445040. E-mail: voropanov.serega@yandex.ru.

The review presents relatively little-known in the Russian literature methods to obtain estimates of output multipliers (final demand multipliers) in terms of minimum information. In these circumstances a researcher has to choose among three following approaches: 1) SBT (Survey-Based Tables) approach - independent development of regional tables on the basis of extensive one-time survey; 2) RIMS (Regional Input-Output Modeling System) approach building estimated regional tables on the basis of national (base or calculated) tables; and 3) SM (Shortcut Methods) approach or methods of minimum information - specific calculations based on traditional statistics information or information that can be derived from traditional statistics, with the least amount of resources for its processing. The author pays special attention to the SM approach: its evolution, critique of presuppositions and results of empirical testing. The study shows that most of the numerous experiments (conducted for different countries, regions, tables of different dimensions and for different years) based on the SM approach give quite acceptable accuracy of multipliers' estimation. On the basis of the theory of positive operators the author obtains two-sided estimates of output multipliers.
\end{abstract}

Keywords: input-output model, regional input-output analysis, output multipliers, final demand multipliers, multipliers' valuation methods, SBT (Survey-Based Tables), RIMS (Regional InputOutput Modeling System), SM (Shortcut Methods).

\section{REFERENCES}

1. Baranov E.F., Kim I.A., Staritsyna E.A. Methodological Questions of the Reconstruction of Input-Output Tables of Russia for 2003 and the Following Years under the Structure of the OKVED - OKPD. Voprosy Statistiki [Statistical Issues], 2011, no. 12, pp. 3-8. (In Russian).

2. Baranov E.F., Kim I.A., Piontkovski D.I., Staritsyna E.A. Methodological Problems of Constructing Systems of Input-Output Tables of Russia in the Classifications of Industries and Products Meet International Standards. Preprint WP2/2013.06. Moscow: National Research University «Higher School of Economics», 2013, 41 p. (In Russian).

3. Baranov E.F., Kim I.A., Piontkovski D.I., Staritsyna E.A. Problems of Constructing Russian Input-Output Tables into the International Class. Ekonomicheskiy Zhurnal VSHEHSE Economic Journal, 2014, no. 1, pp. 7-41. (In Russian).

4. Voropanov S.A. Estimation of Some Characteristics of the Leontief Models: Conditions of Incomplete Information. Estestvennye i Matematicheskie Nauki v Sovremennom Mire [Natural and Mathematical Sciences in the Modern World], 2013, no. 10-11, pp. 92-98. (In Russian).

5. Voropanov S.A. Application of Method of Bilateral Estimation of the Output Multiplier in the Absence of a Complete Input-Output Table: Example of Germany and France. Problemy Prognozirovaniya - Studies on Russian Economic Development, 2015, no. 4, pp. 131-141. (In Russian). 
6. Krasnoselskiy M.A., Vaynikko G.M., Zabreyko P.P., Rutitskiy Ya.B., Stetsenko V.Ya. Approximate Solution of Operator Equations. Moscow, 1969, 456 p. (In Russian).

7. Mathematical Encyclopedia. Available at: http://dic.academic.ru/dic.nsf/enc_mathematics/3696/ОПЕРАЦИОННОЕ (accessed 17 April 2014). (In Russian).

8. Mikheeva N.N. Statistical Estimation of Input-Output Tables for the Russian Far East. Prostranstvennaya Ekonomika = Spatial Economics, 2005, no. 2, pp. 61-79. (In Russian).

9. Nigmatulin R.I., Sayapova A.R., Mazitova L.D. Tables Input-Output of Bashkortostan Republic. ECO - ECO, 2006, no. 3, pp. 90-105. (In Russian).

10. Serebryakov G.R., Uzyakov M.N., Yantovskiy A.A. Interindustry Model of the Economy of the Ivanovo Territory. Problemy Prognozirovaniya - Studies on Russian Economic Development, 2002, no. 5, pp. 64-74. (In Russian).

11. Abramov V.M., Voropanov S.A. Regional Analysis and Forecast under Limited Available Information. Problems and Prospects of Development of the Banking System of the Republic of Tajikistan in the Conditions of Instability of Global Finance: Materials of the Republican Scientific-Practical Conference (Dushanbe, 2014, 15-16 may). Dushanbe, 2014, pp. 133-135.

12. Bekhet H.A. Output, Income and Employment Multipliers in Malaysian Economy: Input-Output Approach. International Business Research, 2011, vol. 4, no. 1, pp. 208-223. DOI: $10.5539 /$ ibr.v4n1p208.

13. Bromley D.W. An Alternative to Input-Output Models: A Methodological Hypothesis. Land Economics, 1972, vol. 48, pp. 125-133. DOI: 10.2307/3145471.

14. Burford R.L., Katz J.L. Regional Input-Output Multipliers without a Full I-O Table: Reply. Annals of Regional Science, 1978, vol. 12, no. 3, pp. 99-102. DOI: 10.1007/ BF01286127.

15. Burford R.L. Regional Input-Output Multipliers without a Full I-O Table. Annals of Regional Science, 1977, vol. 11, no. 3, pp. 21-38. DOI: 10.1007/BF01287766.

16. Burford R.L., Katz J.L. A Method of Estimation of Input-Output Type Output Multipliers when no I-O Model Exists. Journal of Regional Science, 1981, vol. 21, no. 2. DOI: 10.1111/j.1467-9787.1981.tb00691.x.

17. Burford R.L., Katz J.L. An Estimator of Regional Interindustry Multipliers without an I-O Matrix. Business and Economics Section Proceedings of the American Statistical Association, 1977, pp. 453-458.

18. Burford R.L., Katz J.L. Regional Input-Output Multipliers without a Full I-O Table: (Communication). Annals of Regional Science, 1978, vol. 12, no. 2, pp. 105-106. DOI: 10.1007/BF01286115.

19. Burford R.L., Katz J.L. Shortcut Input-Output Multipliers, Alive and Well: Response to Jensen and Hewings. Environment and Planning A, 1985, vol. 17, no. 11, pp. 1541-1549. DOI: $10.1068 /$ a 171541.

20. Drake R.L. A Short Cut to Estimates of Regional Input-Output Multipliers: Methodology and Evaluation. International Regional Science Review, 1976, vol. 1, no. 2, pp. 1-17. DOI: 10.1177/016001767600100201.

21. Harrigan F.J. The Estimation of Input-Output Type Output Multipliers when no Input-Output Model Exists: A Comment. Journal of Regional Science, 1982, vol. 22, no. 3, pp. 375-381. DOI: 10.1111/j.1467-9787.1982.tb00762.x.

22. Input-Output Analysis: Foundations and Extensions. Second Edition. Edited by R.E. Miller, P.D. Blair. Cambridge University Press, 2009, 784 p. DOI: 10.1017/ CBO9780511626982.

23. Jensen R.C., Hewings G.J.D. Shortcut Input-Output Multipliers: A Requiem. Environment and Planning A, 1985, vol. 17, no. 6, pp. 747-759. DOI: 10.1068/a170747. 
24. Jensen R.C., Hewings G.J.D. Shortcut Input-Output Multipliers: The Resurrection Problem (a Reply). Environment and Planning A, 1985, vol. 17, no. 11, pp. 1551-1552. DOI: $10.1068 / \mathrm{a} 171551$.

25. Katz J.L. A Shortcut Method for Computing Final Demand Multipliers for Small Regions: Comment. Environment and Planning A, 1983, vol. 15, no. 4, pp. 551-554. DOI: 10.1068/a150551.

26. Katz J.L., Burford R.L. A Comparison of Estimators of Output Multipliers from Incomplete Input-Output Data. The Annals of Regional Science 1981, vol. 15, no. 2, pp. 39-54. DOI: 10.1007/BF01287438.

27. Katz J.L., Burford R.L. Shortcut Formulas for Output, Income and Employment Multipliers. The Annals of Regional Science, 1985, vol. 19, no. 2, pp. 61-76. DOI: 10.1007/ BF01287578.

28. Katz J.L., Burford R.L. The Estimation of Input-Output Type Output Multipliers when no Input-Output Model Exists: A Reply. Journal of Regional Science, 1982, vol. 22, no. 3, pp. 383-387. DOI: 10.1111/j.1467-9787.1982.tb00763.x.

29. Nelson P.E., Perrin J.S. A Short Cut for Computing Final Demand Multipliers: Some Empirical Results. Land Economics, 1978, vol. 54, no. 1, pp. 82-91. DOI: 10.2307/3146204.

30. Phibbs P.J., Holsman A.J. A Reply to Katz's Comment / Letter to the Editor. Environment and Planning $A$, 1983, vol. 15, no. 4, pp. 555-558. DOI: 10.1068/a150555.

31. Phibbs P.J., Holsman A.J. A Shortcut Method for Computing Final Demand Multipliers for Small Regions. Environment and Planning A, 1980, vol. 12, no. 9, pp. 1001-1008. DOI: $10.1068 /$ a121001.

32. Regional Input-Output Modeling System (RIMS II). Available at: https://www.bea. gov/regional/rims/ (accessed 15 April 2014).

33. Salcedo D. A Short Cut Method for Determining the Impact of an Economic Activity on an Entire Economy. Unpublished Report Office of Information Services. Texas, 1972. Cited by: Jensen R.C., Hewings G.J.D. Shortcut Input-Output Multipliers: A Requiem. Environment and Planning A, 1985, vol. 17, no. 6, pp. 747-759. DOI: 10.1068/ a170747.

34. The World Input-Output Database (WIOD): Contents, Sources and Methods. Edited by M.P. Timmer. WIOD. Working Paper, no. 10, 2012. Available at: http://www.wiod.org/ publications/papers/wiod10.pdf (accessed 06 November 2013). 